\title{
Review Article \\ Levels of Soluble E-Cadherin in Breast, Gastric, and Colorectal Cancers
}

\author{
Ombretta Repetto, ${ }^{1}$ Paolo De Paoli, ${ }^{2}$ Valli De Re, ${ }^{1}$ \\ Vincenzo Canzonieri, ${ }^{3}$ and Renato Cannizzaro ${ }^{4}$ \\ ${ }^{1}$ Facility of Bio-Proteomics, Centro di Riferimento Oncologico (CRO), Aviano National Cancer Institute, \\ Via F. Gallini 2, 33081 Aviano, Italy \\ ${ }^{2}$ Centro di Riferimento Oncologico (CRO), Aviano National Cancer Institute, Via F. Gallini 2, 33081 Aviano, Italy \\ ${ }^{3}$ Pathology Unit, Centro di Riferimento Oncologico (CRO), Aviano National Cancer Institute, Via F. Gallini 2, 33081 Aviano, Italy \\ ${ }^{4}$ Gastroenterology Unit, Centro di Riferimento Oncologico (CRO), Aviano National Cancer Institute, \\ Via F. Gallini 2, 33081 Aviano, Italy
}

Correspondence should be addressed to Valli De Re; vdere@cro.it

Received 3 February 2014; Accepted 18 August 2014; Published 16 September 2014

Academic Editor: Alessandro Luciani

Copyright (c) 2014 Ombretta Repetto et al. This is an open access article distributed under the Creative Commons Attribution License, which permits unrestricted use, distribution, and reproduction in any medium, provided the original work is properly cited.

Soluble E-cadherin is a $80 \mathrm{kDa}$ protein fragment coming from the proteolytic cleavage of the extracellular domain of the full length epithelial cadherin, a molecule involved in cell adhesion/polarity and tissue morphogenesis. In comparison with normal epithelia, cancer cells show a decreased cadherin-mediated intercellular adhesion, and sE-cad levels normally increase in body fluids (blood and urine). This review focuses on soluble E-cadherin in sera of patients affected by three solid cancers (breast, gastric, and colorectal cancers) and how its levels correlate or not with some cancer parameters (e.g., dimension, progression, and localisation). We will describe the main proteomics approaches adopted to measure sE-cad both in vivo and in vitro and the most important findings about its behaviour in cancer dynamics.

\section{The Soluble E-Cadherin}

The E-cadherins (E-cad), or "classical" cadherins of type I, belong to the large family of cadherins, transmembrane or membrane-associated glycoproteins, mediating cell-cell adhesion and playing a pivotal role in epithelial cell behaviour and tissue morphogenesis/remodelling (reviewed in [1-7]). Transcriptional E-cad reprogramming in epithelial cells leads to decreased adhesion and enhanced migration/invasion at the epithelial-to-mesenchymal transition (EMT) during cancer progression [8]. In this context, many cancer researches focus on E-cad expression and its modulation: basic structure of E-cadherin protein, posttranslational processing and maturation, genetic variants, gene expression (activation versus silencing), and transcript content/localization have been widely investigated, together with E-cad interactions with multiprotein complexes and signalling variations associated with alterations of E-cad cell-cell adhesion properties [9-12]. Most epithelial tumors loose E-cad partially or completely through mutation, epigenetic silencing, or increased expression of nonepithelial cadherins (colorectal CRC [13]; gastric cancer (GC) [14-16]; breast [17-22]; and GC and breast cancer [23]), and E-cad downregulation globally correlates with tumor grade and invasion. However, in human breast cancer, Hollestelle et al. [24] recently have observed that E-cad loss was neither causal nor necessary for EMT. At protein level, under pathological conditions, the effects of E-cad-associated genetic changes are usually evaluated in terms of content and localization by in situ hybridization and immunostaining (e.g., $[14,15])$

Other mechanisms potentially influencing E-cad normal functions such as its binding to other proteins include the levels of its phosphorylation together with specific proteolytic events [4]. Indeed, enzymes such as secretases, calpain, and 
caspases may cleave E-cad in its cytoplasmic part, while matrix metalloproteinases (e.g., MMP-2, MMP-3, MMP-7, MMP-9, and MMP-14, stromelysin-1, and matrilysin) and cathepsins (B, L, S), together with other proteases (e.g., disintegrins AAM10 and AAM15 and plasmin), secretases, calpain, and caspases, besides bacterial proteases, can cleave E-cad ectodomain near the plasma membrane and generate a soluble $80 \mathrm{kDa}$ E-cad fragment (sE-cad) released in the extracellular space $[4,25]$.

At present, serum levels of sE-cad are known to increase in patients affected by cancer (e.g., breast, gastric, and colorectal cancers; Table 1) in respect to healthy patients, so that there is a growing interest in sE-cad as "candidate sentinel molecule" in cancer research (reviewed by [2527]). In these cases, the sE-cad levels have been associated with metastatic disease and worse prognosis, and the E-cad cleavage into sE-cad has been linked to malignant adenomacancer progression. However, sE-cad may be also increased due to oxidative stress [28] and production of cytokines involved in inflammation and tumorigenesis $[25,29]$.

Generally, since the first observations in 1990, the global decrease in E-cad in dissociating/metastasising cancer cells was accompanied by an increase in sE-cad fragments in patient sera, so that the first emerging idea was to consider the soluble sE-cad as originating from the rapid turnover of tumor cells and to relate the sE-cad concentration to the tumor size.

Here, we report proteomics applied to the characterization of sE-cad amount in three solid cancers (breast, gastric, and colorectal cancers) and describe the most common techniques adopted since sE-cad discovery. Since sE-cad presence is not only limited to these three pathologies, we also briefly summarized the findings of other works in a recapitulative table (Table 1 ).

\section{Proteomics Approaches Applied to Cadherin Characterization}

2.1. Immunoenzymometric Assay. The protocol by Katayama et al. [27] - modified after Katayama et al. [30]-has been widely adopted to measure serum $\mathrm{sE}$-cad concentrations with a commercially available sandwich ELISA kit. Briefly, as it has been described in Katayama et al. [30], the first monoclonal antibody (the human E-cadherin-1 monoclonal antibody, HECD-1, raised against the cad extracellular domain) is coated onto microtiter plate wells and creates the solid phase. Nonspecific binding is blocked by a buffer. Serum samples from patients and standard solutions are then incubated in the microtiter plate wells. The second monoclonal antibody, SHE 13-1, labelled with peroxidase is added. During incubation, human E-cad molecule is trapped by the two monoclonal antibodies as a sandwich, so that the technique is also reported as sandwich-type immunoenzymometric assay (IEMA). The reaction between the peroxidase and substrate solution results in colour development with intensities proportional to the concentration of human sE-cad in serum samples and standards. The absorbance of the developed colour is measured at $450 \mathrm{~nm}$. Accurate sample concentrations of human E-cad are determined by comparing specific absorbance with those obtained from the standards plotted on a standard curve. The authors advised the use of serum for routine in cadherin assay, since dissolved $\mathrm{Ca}^{2+}$ is not artificially removed by chelation and a coexistent $\mathrm{Ca}^{2+}$ is necessary to stabilize the structure of sE-cad immunoreactive with those monoclonal antibodies.

2.2. Western Blotting (WB). In most reports, in patients, the sE-cad amount is also evaluated with WB after protein separation by one-dimensional acrylamide gel electrophoresis (1-DE), and it can be compared with the full length E-cad expression, which in turn is analysed by immunostaining in situ. WB analyses reveal the presence of multiple bands, among which are the full length E-cad at $120 \mathrm{kDa}$ and the sEcad at $80 \mathrm{kDa}$.

2.3. Reverse Phase Protein Array (RPPA). Another targeted approach was used by Perez-Rivas et al. [31] to analyse the expression levels of 42 selected serum soluble proteins using the reverse phase protein array technology (RPPA) (described by [32]). The RPPA represents a high-throughput and sensitive technology platform for quantitative and multiplexed immunoassays, in which small amounts of cell lysates or fluids (e.g., serum and urine) are immobilized on individual spots onto a capturing surface called microarray that is then incubated with a single specific antibody to detect the expression of the target protein across many samples. Detection is performed using either a primary or a secondary labeled antibody by chemiluminescent, fluorescent, or colorimetric assays, the array is then imaged, and the obtained data are quantified.

\section{Soluble E-Cadherin in Breast Cancer}

In BC patients, first studies started in 2005 when Hofmann and colleagues measured sE-cad levels in sera of 133 patients before and after neoadjuvant chemotherapy with an enzymebased immunoassay technique, and they positively correlated them with the pre- and posttherapeutic tumor size as well as the disease-free interval [33]. In the same year, Dollé et al. [34] evaluated by 1-DE and WB (anti-HECD-1) the release of sE-cad in the media of MCF-7/AZ BC cells grown in presence or absence of the nerve growth factor (NGF), a small secreted protein that is important for the development and survival of certain target neurons, and their results supported a relation between sE-cad levels and the $\mathrm{BC}$ cell acquisition of an invasive phenotype. In order to identify markers for BC patient response to surgery, the following analyses were addressed to characterize the differential serum proteomes "before versus after surgery" by RPPA [31]. Among the 45 polyclonal antibodies, $s E$-cad resulted to be increased after surgery in sera of invasive tumor patients as compared with those of healthy women and patients with noninvasive tumors. The potential value of sE-cad as marker to predict response(s) to or to assess prognosis after preoperative systemic chemotherapy (PST) was further tested in sera of 108 patients with locally advanced BC using a commercially 


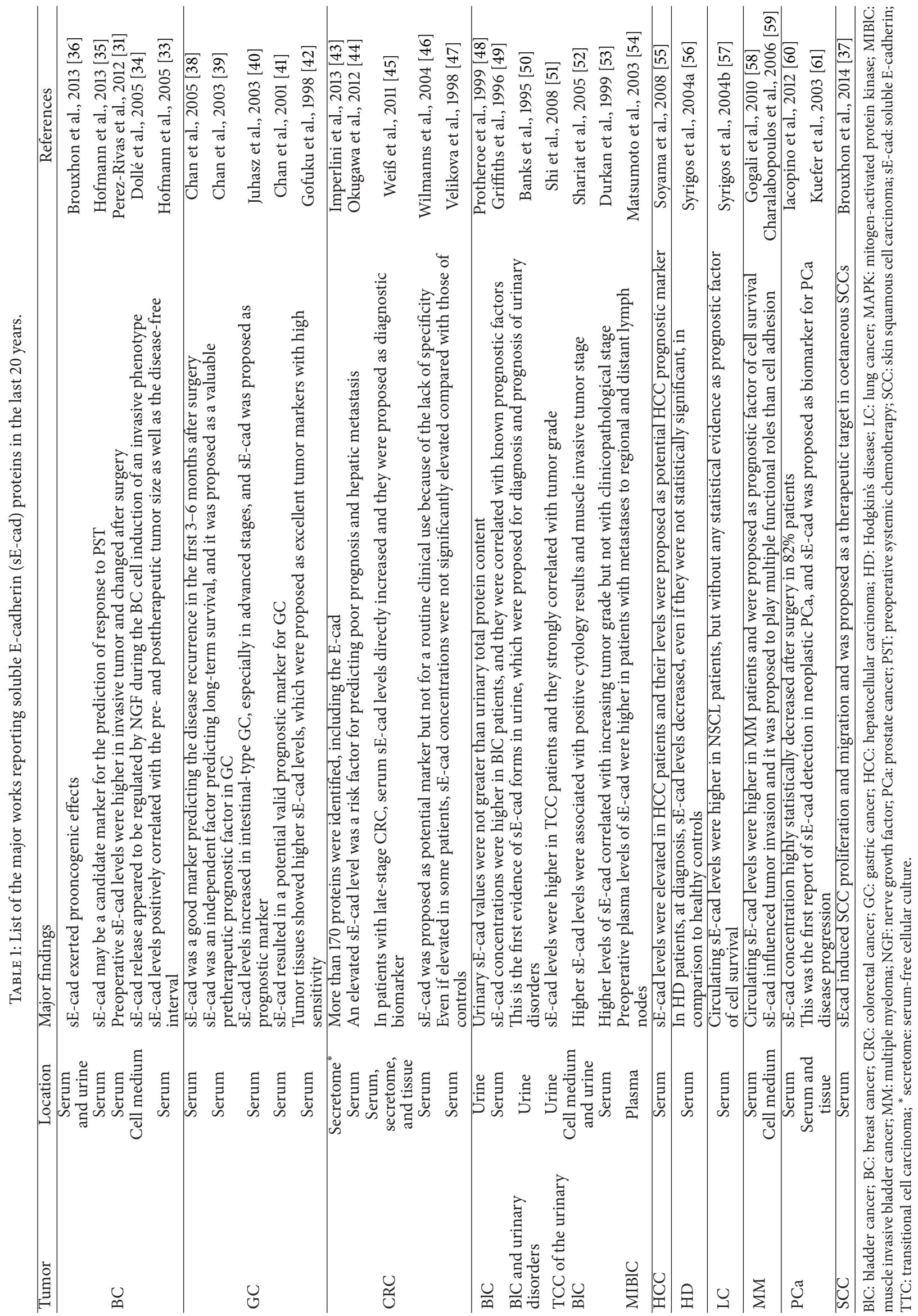


available ELISA kit [35]. Soluble E-cad level was significantly lower in sera of preoperatively treated BC patients undergoing PST with pathological complete response (pCR), suggesting a putative role of $\mathrm{sE}$-cad as a predictive marker for $\mathrm{BC}$ patients for chemotherapy response. In the same year, Brouxhon et al. [36,37] explored whether endogenous sE-cad levels (i) were overexpressed in HER2+ human and mouse BC specimens and tumors and cell culture systems of human triple-negative $\mathrm{BC}$ (TNBC), which does not express the genes for estrogen and progesterone receptors, and Her2/neu, and (ii) exerted prooncogenic effects via modulation of the HERPI3K/Akt/mTOR-IAP axis and/or synergism with the HER ligand EGF. Immunoprecipitation assays were performed after cell lysate incubation with EGFR/HER1, HER2, HER3, HER4, or E-cad ectodomain-specific antibodies, and the immune complexes were then separated through acrylamide 1-DE, while sE-cad levels were quantified using human Ecadherin Quantikine ELISA Kits in sera, urines, and cell conditioned media. Globally, sE-cad protein expression levels increased in the all tested experimental systems, sE-cad contributed via HER family members to enhance MAPK, $\mathrm{PI} 3 \mathrm{~K} / \mathrm{Akt} / \mathrm{mTOR}$, and IAP signaling, it played prooncogenic functions in both HER2+ and TNBC cell lines, and in vitro it acted together with the EGF ligand to promote $\mathrm{BC}$ proliferation, migration, and invasion.

\section{Soluble E-Cadherin in Gastric Cancer}

In GC patients, sE-cad fragments were firstly investigated by Gofuku et al. [42] who compared the amount of serum sE-cad in 81 GC patients: $s E$-cad levels were measured [27], and they resulted to be significantly higher in GC patients than in healthy controls, with the highest sE-cad increase being revealed in the GC tissues having a partially reduced expression of the full length E-cad. In this work, sE-cad levels decreased after tumor removal by surgery. This same assay was further adopted by Chan et al. [41], who found significantly higher sE-cad concentrations in GC patients $(n r=$ 116), correlating them with tumor size and carcinoembryonic antigen (CEA) amount. The same team further correlated serum levels of sE-cad with protein expression in a trial of 116 patients [39]: the sE-cad was found as an independent factor predicting long-term survival, with $90 \%$ of patients with a serum level of sE-cad $>10,000 \mathrm{ng} / \mathrm{mL}$ having a survival time $<$ 3 years. In the same year, by comparing GC patients classified as intestinal-type (51\%) to those with a diffuse-type (49\%) according to Laurén, Juhasz et al. [40] showed that serum sE-cad levels increased in intestinal-type GC, especially in advanced stages, whereas in the diffuse-type sE-cad levels decreased in advanced and metastasized cases. The sE-cad concentrations were thus proposed to be interpreted along with Laurén classification and to represent a negative prognostic marker in the only intestinal GC type. Blood samples of 69 GC patients who underwent curative surgery were assayed prospectively (preoperatively, 1 month after surgery and every 3 months thereafter) for $\mathrm{sE}$-cad and compared to CEA using the method described by Katayama et al. $[27,38]$ : this study documented the use of sE-cad levels in predicting and detecting recurrent GC in the first 36 months after patients undergo curative surgery, with a sensitivity significantly better compared to that one of CEA with the reported cut-off level. The importance of these findings to stratify patients into "low-" and "high-" risk groups for consideration of more aggressive adjuvant therapy and vigilant surveillance in the hope of improved survival was evidenced by the authors.

\section{Soluble E-Cadherin in Colorectal Cancer}

Assays for the detection of sE-cad in blood samples of CRC patients were firstly performed by Velikova et al. [47] with the IEMA assay: in CRC patients versus healthy subjects, though elevated in some patients, sE-cad concentrations were not significantly elevated compared with those of the control group, which was different from what was observed for serum concentrations of intercellular adhesion molecule1 (ICAM-1) and vascular cell adhesion molecule-1 (VCAM-1), which were significantly elevated. Potential marker characteristics for sE-cad in CRC were further evidenced by Wilmanns et al. [46], who however excluded a routine clinical use due to a lack of specificity. In particular, sE-cad concentrations were increased not only in CRC patients, but also in benign disease controls, and they correlated with the primary tumour or " $\mathrm{T}$ stage" (UICC-TNM classification) and serum CEA in case of existing liver metastases. In 2007, concomitant to the development of protocols for the total proteins released by cells, also known as "secretome," Diehl et al. [62] presented twodimensional acrylamide gel electrophoresis (2-DE) maps for the secretome released by SW620 human CRC cells in vitro. Total proteins were compared with the secreted ones by differential in gel electrophoresis (DIGE) in order to search for the secretome-specific proteins of CRC cells. Among the identified secretome-specific spots, there was the sE-cad (accession number: gi|6682963; experimental versus theoretical PI and MW: 4.2/4.6 and >85/99.7). However, the prognostic value of sE-cad level has not been clearly demonstrated yet. In a further study from 186 CRC patients, serum sE-cad levels were related to clinicopathological findings [44]. Preoperative elevated sE-cad levels were found to be associated with poor prognosis in CRC and, in particular, sE-cad measures were thought to provide valuable information for predicting prognosis in patients with hepatic metastasis. Previously, Weiß et al. [45] compared sE-cad levels in sera from patients with CRC, colorectal adenoma, inflammatory bowel disease, and familial adenomatous polyposis, and they found a significant increase in sE-cad load in patients with late-stage CRC (Stages III and IV). In individual patients with late-stage CRC, sE-cad serum levels were proposed to directly reflect their disease status over time. These results suggested a potential application of sE-cad as an alternative diagnostic biomarker for monitoring disease particularly in patients with CAE negative tumors.

\section{Conclusion}

All the analyses hereby described the evidence that the serum $\mathrm{sE}$-cad levels generally increase in many cases concomitant to 
the decrease in full length E-cad expression in tissues, sE-cad, thus behaving like a putative "disease sentinel." Because of its stability in serum, sE-cad accumulating in the blood may serve as sensitive indicator of tumor-associated proteolysis and potential candidate for an early disease detection. However, when the sE-cad decrease in patient sera is associated with particular cancer characteristics (e.g., stage, size, and localization), therapy (e.g., before or after therapy; before or after surgery), or other clinical parameters (e.g., levels of markers such as CEA or other adhesion molecules), it becomes hard to generalize findings and extremely difficult to propose the sE-cad protein as prognostic marker in clinical trials. The sE-cad molecule like CEA does not appear to be adequate for screening purposes due to a lack of specificity and sensibility; however, they both could be tested in association with monitor therapies. Proteomics offers a powerful approach to go further with sE-cad characterization. For instance, it may be tempting to analyze sE-cad levels in parallel with those of other key molecules or markers and to characterize sE-cad associated with $\mathrm{CDH} 1$ mutations at gene level both in vivo and in vitro or to expand knowledge about the candidate molecules triggering cleavage of the full length E-cad into its soluble form. Moreover, the parallel characterization of the secretome will help to better decipher the surprisingly abundant subset of subproteome, which is partly constituted by ectodomains of membrane proteins cleaved from the cell surface such as sE-cad.

\section{Abbreviations}

1-DE: One-dimensional acrylamide gel electrophoresis

2-DE: Two-dimensional acrylamide gel electrophoresis

BlC: Bladder cancer

BC: $\quad$ Breast cancer

CEA: Carcinoembryonic antigen

CRC: Colorectal cancer

DIGE: Difference gel electrophoresis

ELISA: Enzyme linked immunospecific assay

GC: $\quad$ Gastric cancer

HD: Hodgkin's disease

HER: Human epidermal growth factor receptor

IEMA: Immunoenzymometric assay

IGF-1R: Insulin-like growth factor-1 receptor

MAPK: Mitogen-activated protein kinase

MIBlC: Muscle invasive bladder cancer

MM: Multiple myeloma

MMP: Matrix metalloproteinase

MS: $\quad$ Mass spectrometry

mTOR: Mammalian target of rapamycin

NCT: Neoadjuvant chemotherapy

NSCLC: Non-small cell lung cancer

PAGE: Polyacrylamide gel electrophoresis

PCa: Prostate cancer

PI3K: Phosphatidylinositol 3-kinase

PST: $\quad$ Preoperative systemic chemotherapy
SCC: $\quad$ Skin squamous cell carcinoma

SCLC: $\quad$ Small cell lung cancer

SDS: $\quad$ Sodium dodecyl sulphate

sE-cad: Soluble E-cadherin

sICAM-1: Soluble intercellular adhesion molecule-1

sVCAM-1: Soluble vascular cell adhesion molecule-1

TTC: Transitional cell carcinoma

TURBT: Transurethral resection of bladder tumor

WB: Western blotting.

\section{Conflict of Interests}

The authors declare that there is no conflict of interests regarding the publication of this paper.

\section{Acknowledgment}

The work was supported by the Italian Association for Cancer Research (AIRC no. 10266).

\section{References}

[1] W. J. Nelson, D. J. Dickinson, and W. I. Weis, "Roles of cadherins and catenins in cell-cell adhesion and epithelial cell polarity," Progress in Molecular Biology and Translational Science, vol. 116, pp. 3-23, 2013.

[2] F. Twiss and J. De Rooij, "Cadherin mechanotransduction in tissue remodeling," Cellular and Molecular Life Sciences, vol. 70, no. 21, pp. 4101-4116, 2013.

[3] E. Tsanou, D. Peschos, A. Batistatou, A. Charalabopoulos, and K. Charalabopoulos, "The E-cadherin adhesion molecule and colorectal cancer. A global literature approach," Anticancer Research, vol. 28, no. 6, pp. 3815-3826, 2008.

[4] F. van Roy and G. Berx, "The cell-cell adhesion molecule Ecadherin," Cellular and Molecular Life Sciences, vol. 65, no. 23, pp. 3756-3788, 2008.

[5] J. M. Halbleib and W. J. Nelson, "Cadherins in development: cell adhesion, sorting, and tissue morphogenesis," Genes and Development, vol. 20, no. 23, pp. 3199-3214, 2006.

[6] W.-H. Lien, O. Klezovitch, and V. Vasioukhin, "Cadherincatenin proteins in vertebrate development," Current Opinion in Cell Biology, vol. 18, no. 5, pp. 499-506, 2006.

[7] B. M. Gumbiner, "Regulation of cadherin-mediated adhesion in morphogenesis," Nature Reviews Molecular Cell Biology, vol. 6, no. 8, pp. 622-634, 2005.

[8] A. Gheldof and G. Berx, "Cadherins and epithelial-tomesenchymal transition," Progress in Molecular Biology and Translational Science, vol. 116, pp. 317-336, 2013.

[9] M. Canel, A. Serrels, M. C. Frame, and V. G. Brunton, "Ecadherin-integrin crosstalk in cancer invasion and metastasis," Journal of Cell Science, vol. 126, no. 2, pp. 393-401, 2013.

[10] F. J. Rodriguez, L. J. Lewis-Tuffin, and P. Z. Anastasiadis, "E-cadherin's dark side: possible role in tumor progression," Biochimica et Biophysica Acta: Reviews on Cancer, vol. 1826, no. 1, pp. 23-31, 2012.

[11] A. Jeanes, C. J. Gottardi, and A. S. Yap, "Cadherins and cancer: how does cadherin dysfunction promote tumor progression?" Oncogene, vol. 27, no. 55, pp. 6920-6929, 2008. 
[12] R. B. Hazan, R. Qiao, R. Keren, I. Badano, and K. Suyama, "Cadherin switch in tumor progression," Annals of the New York Academy of Sciences, vol. 1014, pp. 155-163, 2004.

[13] A. M. Todosi, M. M. Gavrilescu, G. M. Anitei, B. Filip, and V. Scripcariu, "Colon cancer at the molecular level-usefulness of epithelial-mesenchymal transition analysis," Revista MedicoChirurgicala a Societatii de Medici si Naturalisti din Iasi's, vol. 116, pp. 1106-1111, 2012.

[14] J. Figueiredo, O. Söderberg, J. Simões-Correia, K. Grannas, G. Suriano, and R. Seruca, "The importance of E-cadherin binding partners to evaluate the pathogenicity of E-cadherin missense mutations associated to HDGC," European Journal of Human Genetics, vol. 21, no. 3, pp. 301-309, 2013.

[15] J. Simões-Correia, J. Figueiredo, R. Lopes et al., "E-cadherin destabilization accounts for the pathogenicity of missense mutations in hereditary diffuse gastric cancer," PLoS ONE, vol. 7, no. 3, Article ID e33783, 2012.

[16] K.-F. Becker, M. J. Atkinson, U. Reich et al., "E-cadherin gene mutations provide clues to diffuse type gastric carcinomas," Cancer Research, vol. 54, no. 14, pp. 3845-3852, 1994.

[17] K. Chu, K. M. Boley, R. Moraes, S. H. Barsky, and F. M. Robertson, "The paradox of E-cadherin: role in response to hypoxia in the tumor microenvironment and regulation of energy metabolism," Oncotarget, vol. 4, no. 3, pp. 446-462, 2013.

[18] S. Baranwal and S. K. Alahari, "Molecular mechanisms controlling E-cadherin expression in breast cancer," Biochemical and Biophysical Research Communications, vol. 384, no. 1, pp. 6-11, 2009.

[19] T. L. Mastracci, S. Tjan, A. L. Bane, F. P. O’Malley, and I. L. Andrulis, "E-cadherin alterations in atypical lobular hyperplasia and lobular carcinoma in situ of the breast," Modern Pathology, vol. 18, no. 6, pp. 741-751, 2005.

[20] G. Berx, A.-M. Cleton-Jansen, K. Strumane et al., "E-cadherin is inactivated in a majority of invasive human lobular breast cancers by truncation mutations throughout its extracellular domain," Oncogene, vol. 3, no. 9, pp. 1919-1925, 1996.

[21] Y.-E. Joo, J.-S. Rew, S.-K. Choi, H.-S. Bom, C.-S. Park, and S.J. Kim, "Expression of E-cadherin and catenins in early gastric cancer," Journal of Clinical Gastroenterology, vol. 35, no. 1, pp. 35-42, 2002.

[22] G. Berx, A.-M. Cleton-Jansen, F. Nollet et al., "E-cadherin is a tumour/invasion suppressor gene mutated in human lobular breast cancers," EMBO Journal, vol. 14, no. 24, pp. 6107-6115, 1995.

[23] G. Berx, K. F. Becker, H. Höfler, and F. van Roy, "Mutations of the human E-cadherin (CDH1) gene," Human Mutation, vol. 12, pp. 226-237, 1998.

[24] A. Hollestelle, J. K. Peeters, M. Smid et al., "Loss of E-cadherin is not a necessity for epithelial to mesenchymal transition in human breast cancer," Breast Cancer Research and Treatment, vol. 138, no. 1, pp. 47-57, 2013.

[25] M. M. Grabowska and M. L. Day, "Soluble E-cadherin: more than a symptom of disease," Frontiers in Bioscience, vol. 17, no. 5, pp. 1948-1964, 2012.

[26] O. de Wever, L. Derycke, A. Hendrix et al., "Soluble cadherins as cancer biomarkers," Clinical and Experimental Metastasis, vol. 24, no. 8, pp. 685-697, 2007.

[27] M. Katayama, S. Hirai, K. Kamihagi, K. Nakagawa, M. Yasumoto, and I. Kato, "Soluble E-cadherin fragments increased in circulation of cancer patients," British Journal of Cancer, vol. 69 , no. 3, pp. $580-585,1994$.
[28] A. R. Parrish, J. M. Catania, J. Orozco, and A. J. Gandolfi, "Chemically induced oxidative stress disrupts the Ecadherin/catenin cell adhesion complex," Toxicological Sciences, vol. 51, no. 1, pp. 80-86, 1999.

[29] A. J. Karayiannakis, K. N. Syrigos, A. Savva, A. Polychronidis, G. Karatzas, and C. Simopoulos, "Serum E-cadherin concentrations and their response during laparoscopic and open cholecystectomy," Surgical Endoscopy and Other Interventional Techniques, vol. 16, no. 11, pp. 1551-1554, 2002.

[30] M. Katayama, K. Kamihagi, S. Hirai et al., "Urinary laminin fragments as a tumour marker potentially reflecting basement membrane destruction," British Journal of Cancer, vol. 65, no. 4, pp. 509-514, 1992.

[31] L. G. Perez-Rivas, J. M. Jerez, C. E. Fernandez-De Sousa et al., "Serum protein levels following surgery in breast cancer patients: a protein microarray approach," International Journal of Oncology, vol. 41, no. 6, pp. 2200-2206, 2012.

[32] C. Mueller, L. A. Liotta, and V. Espina, "Reverse phase protein microarrays advance to use in clinical trials," Molecular Oncology, vol. 4, no. 6, pp. 461-481, 2010.

[33] G. Hofmann, N. Dandachi, M. Balic et al., "Quantitative measurement of soluble E-cadherin in sera of breast cancer patients treated with neoadjuvant anthracycline-based chemotherapy and its predictive and prognostic value," Journal of Clinical Oncology, vol. 23, supplement 768, Meeting abstracts, no. 16, 2005.

[34] L. Dollé, M.-J. Oliveira, E. Bruyneel, H. Hondermarck, and M. Bracke, "Nerve Growth Factor mediates its pro-invasive effect in parallel with the release of a soluble E-cadherin fragment from breast cancer MCF-7/AZ cells," Journal of Dairy Research, vol. 72, pp. 20-26, 2005.

[35] G. Hofmann, M. Balic, N. Dandachi et al., "The predictive value of serum soluble E-cadherin levels in breast cancer patients undergoing preoperative systemic chemotherapy," Clinical Biochemistry, vol. 46, no. 15, pp. 1585-1589, 2013.

[36] S. M. Brouxhon, S. Kyrkanides, X. Teng et al., "Soluble-Ecadherin activates HER and IAP family members in HER2+ and TNBC human breast cancers," Molecular Carcinogenesis, 2013.

[37] S. M. Brouxhon, S. Kyrkanides, X. Teng et al., "Soluble Ecadherin: a critical oncogene modulating receptor tyrosine kinases, MAPK and PI3K/Akt/mTOR signaling," Oncogene, vol. 33, no. 2, pp. 225-235, 2014.

[38] A. O. O. Chan, K.-M. Chu, S. K. Lam et al., "Early prediction of tumor recurrence after curative resection of gastric carcinoma by measuring soluble E-cadherin," Cancer, vol. 104, no. 4, pp. 740-746, 2005.

[39] A. O.-O. Chan, K.-M. Chu, S.-K. Lam et al., "Soluble E-cadherin is an independent pretherapeutic factor for long-term survival in gastric cancer," Journal of Clinical Oncology, vol. 21, no. 12, pp. 2288-2293, 2003.

[40] M. Juhasz, M. P. A. Ebert, H. U. Schulz et al., "Dual role of serum soluble E-cadherin as a biological marker of metastatic development in gastric cancer," Scandinavian Journal of Gastroenterology, vol. 38, no. 8, pp. 850-855, 2003.

[41] A. O. O. Chan, S. K. Lam, K. M. Chu et al., "Soluble E-cadherin is a valid prognostic marker in gastric carcinoma," Gut, vol. 48, no. 6, pp. 808-811, 2001.

[42] J. Gofuku, H. Shiozaki, Y. Doki et al., "Characterization of soluble E-cadherin as a disease marker in gastric cancer patients," British Journal of Cancer, vol. 78, no. 8, pp. 1095-1101, 1998. 
[43] E. Imperlini, I. Colavita, M. Caterino et al., "The secretome signature of colon cancer cell lines," Journal of Cellular Biochemistry, vol. 114, no. 11, pp. 2577-2587, 2013.

[44] Y. Okugawa, Y. Toiyama, Y. Inoue et al., "Clinical significance of serum soluble E-cadherin in colorectal carcinoma," Journal of Surgical Research, vol. 175, no. 2, pp. e67-e73, 2012.

[45] J. V. Weiß, S. Klein-Scory, S. Kübler et al., "Soluble E-cadherin as a serum biomarker candidate: elevated levels in patients with late-stage colorectal carcinoma and FAP," International Journal of Cancer, vol. 128, no. 6, pp. 1384-1392, 2011.

[46] C. Wilmanns, J. Grossmann, S. Steinhauer et al., "Soluble serum E-cadherin as a marker of tumour progression in colorectal cancer patients," Clinical \& Experimental Metastasis, vol. 21, no. 1, pp. 75-78, 2004.

[47] G. Velikova, R. E. Banks, A. Gearing et al., "Serum concentrations of soluble adhesion molecules in patients with colorectal cancer," British Journal of Cancer, vol. 77, no. 11, pp. 1857-1863, 1998.

[48] A. S. Protheroe, R. E. Banks, M. Mzimba et al., "Urinary concentrations of the soluble adhesion molecule E-cadherin and total protein in patients with bladder cancer," The British Journal of Cancer, vol. 80, no. 1-2, pp. 273-278, 1999.

[49] T. R. L. Griffiths, I. Brotherick, R. I. Bishop et al., "Cell adhesion molecules in bladder cancer: soluble serum E-cadherin correlates with predictors of recurrence," The British Journal of Cancer, vol. 74, no. 4, pp. 579-584, 1996.

[50] R. E. Banks, W. H. Porter, P. Whelan, P. H. Smith, and P. J. Selby, "Soluble forms of the adhesion molecule E-cadherin in urine," Journal of Clinical Pathology, vol. 48, no. 2, pp. 179-180, 1995.

[51] B. Shi, V. Laudon, S. Yu, D. Dong, Y. Zhu, and Z. Xu, "Ecadherin tissue expression and urinary soluble forms of Ecadherin in patients with bladder transitional cell carcinoma," Urologia Internationalis, vol. 81, no. 3, pp. 320-324, 2008.

[52] S. F. Shariat, K. Matsumoto, R. Casella, W. Jian, and S. P. Lerner, "Urinary levels of soluble E-cadherin in the detection of transitional cell carcinoma of the urinary bladder," European Urology, vol. 48, no. 1, pp. 69-76, 2005.

[53] G. C. Durkan, I. Brotherick, and J. K. Mellon, "The impact of transurethral resection of bladder tumour on serum levels of soluble E-cadherin," BJU International, vol. 83, no. 4, pp. 424428, 1999.

[54] K. Matsumoto, S. F. Shariat, R. Casella, T. M. Wheeler, K. M. Slawin, and S. P. Lerner, "Preoperative plasma soluble Ecadherin predicts metastases to lymph nodes and prognosis in patients undergoing radical cystectomy," Journal of Urology, vol. 170, no. 6, pp. 2248-2252, 2003.

[55] A. Soyama, S. Eguchi, M. Takatsuki et al., "Significance of the serum level of soluble E-cadherin in patients with HCC," Hepato-Gastroenterology, vol. 55, no. 85, pp. 1390-1393, 2008.

[56] K. N. Syrigos, E. Salgami, A. J. Karayiannakis, N. Katirtzoglou, E. Sekara, and P. Roussou, "Prognostic significance of soluble adhesion molecules in Hodgkin's disease," Anticancer Research, vol. 24, no. 2, pp. 1243-1247, 2004.

[57] K. N. Syrigos, K. J. Harrington, A. J. Karayiannakis, N. Baibas, N. Katirtzoglou, and P. Roussou, "Circulating soluble E-cadherin levels are of prognostic significance in patients with multiple myeloma," Anticancer Research, vol. 24, no. 3, pp. 2027-2031, 2004.

[58] A. Gogali, K. Charalabopoulos, I. Zampira et al., "Soluble adhesion molecules E-cadherin, intercellular adhesion molecule-1, and E-selectin as lung cancer biomarkers," Chest, vol. 138, no. 5, pp. 1173-1179, 2010.
[59] K. Charalabopoulos, A. Gogali, Y. Dalavaga et al., "The clinical significance of soluble E-cadherin in nonsmall cell lung cancer," Experimental Oncology, vol. 28, no. 1, pp. 83-85, 2006.

[60] F. Iacopino, F. Pinto, A. Bertaccini et al., "Soluble E-cadherin and IL- 6 serum levels in patients affected by prostate cancer before and after prostatectomy," Oncology Reports, vol. 28, no. 1, pp. 370-374, 2012.

[61] R. Kuefer, M. D. Hofer, J. E. Gschwend et al., "The role of an $80 \mathrm{kDa}$ fragment of E-cadherin in the metastatic progression of prostate cancer," Clinical Cancer Research, vol. 9, no. 17, pp. 6447-6452, 2003.

[62] H. C. Diehl, K. Stühler, S. Klein-Scory et al., "A catalogue of proteins released by colorectal cancer cells in vitro as an alternative source for biomarker discovery," Proteomics: Clinical Applications, vol. 1, no. 1, pp. 47-61, 2007. 

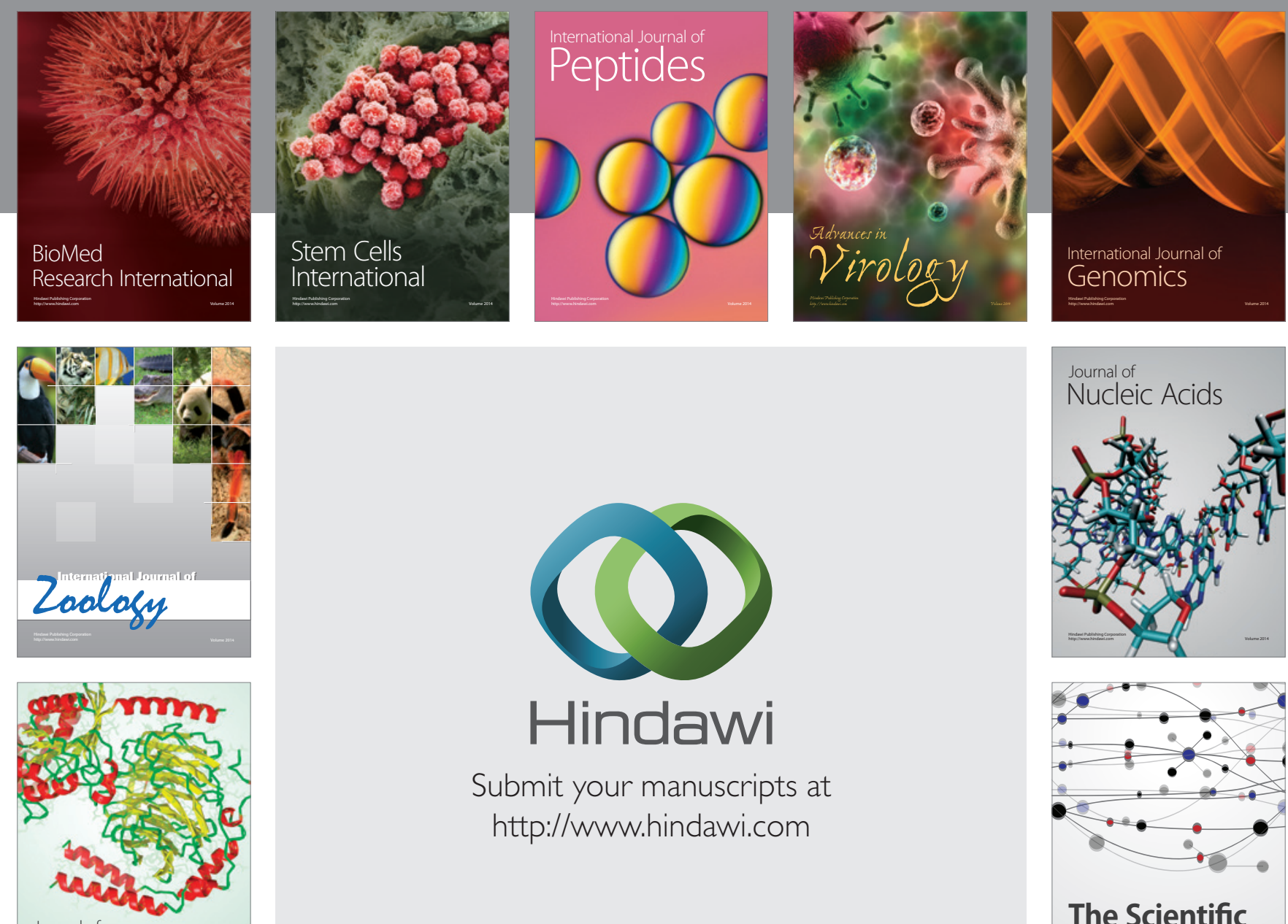

Submit your manuscripts at

http://www.hindawi.com

Journal of
Signal Transduction
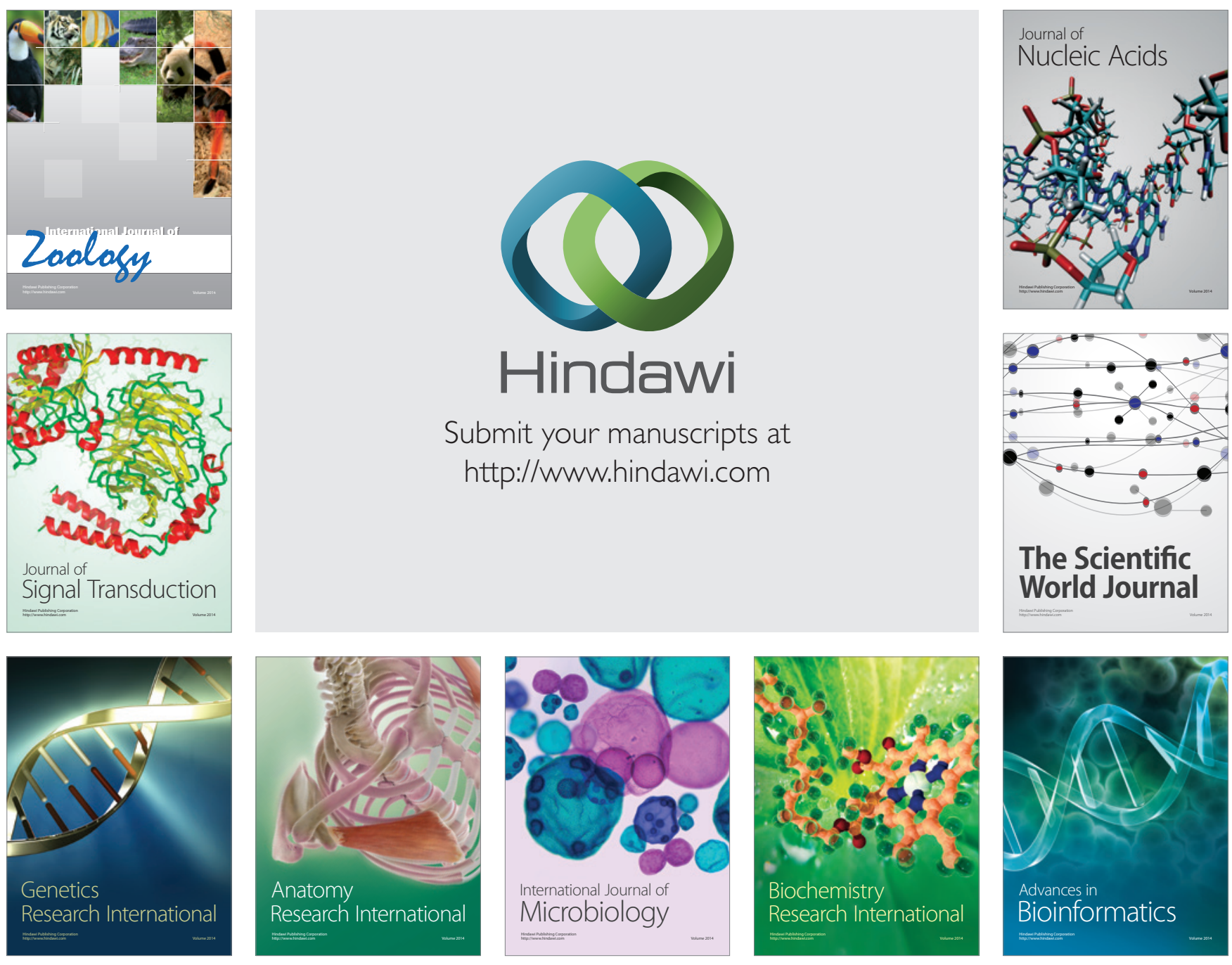

The Scientific World Journal
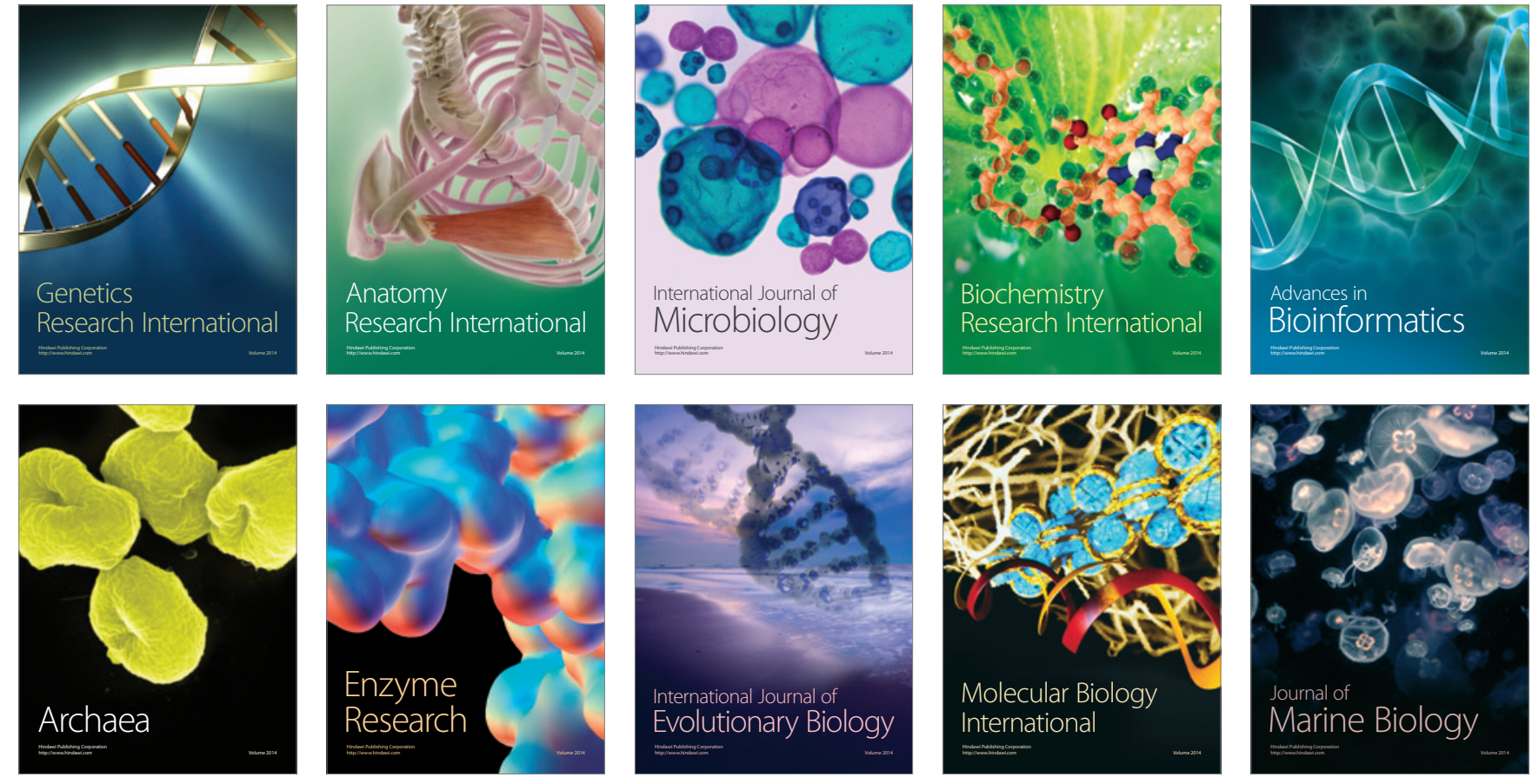